\title{
SISTEMAS PRODUTO-SERVIÇO: REFERENCIAL TEÓRICO E DIREÇÕES PARA FUTURAS PESQUISAS
}

\section{PRODUCT-SERVICE SYSTEM: THEORETICAL FRAMEWORK AND FUTURE RESEARCH DIRECTIONS}

\author{
Miriam Borchardt* miriamb@unisinos.br \\ Miguel Afonso Sellitto* sellitto@unisinos.br \\ Giancarlo Medeiros Pereira* gian@unisinos.br \\ * Universidade do Vale do Rio dos Sinos (UNISINOS)
}

Resumo: Neste artigo, caracterizado como teórico e exploratório, assume-se uma perspectiva crítica de análise sobre o tema product-service system (PSS - Sistemas Produto-Serviço). O PSS é um sistema integrado de bens e serviços que visa oferecer soluções completas e integradas a consumidores de bens de base tecnológica. $O$ objetivo deste trabalho é construir um marco referencial acerca dos elementos essenciais a serem considerados na concepção e gestão de um PSS, focalizando as diferentes abordagens verificadas na literatura. O PSS teve sua origem na sustentabilidade ambiental: a partir de um uso mais intensivo dos produtos, por meio de aluguel, compartilhamento ou multiuso, os recursos físicos teriam seu uso maximizado; tal abordagem tem como premissa a existência de um gerenciamento adequado desses bens físicos. Posteriormente, uma abordagem com foco na sustentabilidade econômica passou a ser considerada: a entrega de soluções no lugar de bens físicos gera relações de longo prazo com os consumidores e diversos parceiros. Foram identificadas, também, as categorias de PSS, os elementos a serem considerados no projeto de um PSS, as vantagens e os desafios decorrentes da aplicação do mesmo. Pretende-se que essa pesquisa teórica venha servir de base para posteriores estudos de casos exploratórios e um survey sobre o tema.

Palavras-chave: Sistemas Produto-serviço. Servitização, Gestão de operações

Abstract: This theoretical and exploratory paper aims to build a critical analysis on product-service system (PSS). The PSS is an integrated system of products and services whose purpose is to offer solutions for the customers instead of only products or services. The goal of the research is to achieve some better understanding about the essential elements that are to be taken into account in conceiving such PSS, focusing on different approaches. The origin of the PSS is linked to environmental sustainability: the more intensive use of the product, by renting, sharing or pooling, leading to the optimization in the use of the physical goods. Later on, an approach with an economic view has been considered too: providing solutions instead of only physical products, promoting, this way, the desirable long-term relationship with customers and partners. It was identified in this paper not only the PSS categories and factors to be considered in its design, but also its advantages and barriers. Finally, this theoretical research is intended to be the basis for further exploratory case studies and survey related with PSS.

Key-words: Product-service system. Servitization. Operations management. 


\section{INTRODUÇÃO}

Manter o foco na produção de produtos físicos pode não ser mais suficiente para garantir vantagem competitiva em algumas indústrias, principalmente àquelas ligadas à manufatura de bens tecnológicos. Para Borchardt et al. (2008a), bens manufaturados são considerados de base tecnológica se o nível tecnológico presente for decisivo para o resultado do cliente final, tal como ocorre em equipamentos eletrônicos para uso industrial ou máquinas operatrizes. A produção de itens manufaturados com serviços associados ao mesmo vem ganhando espaço e expande a visão de operações para um contexto amplo que engloba operações de serviços e de manufatura conjuntamente (GEBAUER, 2008).

Nesse sentido, Oliva e Kallenberg (2003) sugerem três abordagens na associação entre bens manufaturados e serviços especializados. A primeira se apóia em argumentos econômicos: receitas podem ser geradas por serviços associados a produtos de longo ciclo de vida; serviços, em geral, têm uma margem de lucro maior e são mais resistentes aos ciclos econômicos do que os produtos manufaturados. A segunda abordagem versa sobre a questão tecnológica: consumidores estão demandando produtos de tecnologia mais complexa, que requerem serviços cada vez mais especializados. A terceira diz respeito à competição: serviços são mais dependentes de talentos e mais difíceis de imitar, tornando-se fonte de vantagem competitiva.

A integração de bens e serviços trás consigo desafios com relação ao projeto do produto, que passa a ser considerado como um pacote ou oferta de soluções. Os processos produtivos, agora de bens e serviços, também precisam ser repensados. Da mesma forma, a entrega do produto apresenta escopo ampliado. $\mathrm{O}$ ciclo de vida do produto também pode ser modificado considerando-se que o produto passa a englobar serviços associados (YANG et al., 2009). Aspectos intangíveis dos serviços somam-se aos aspectos tangíveis dos bens manufaturados, criando ambiente propício para o surgimento de relações duradouras entre parceiros. Para Yanamandram e White (2006), construída a confiança entre as partes, a tendência é que surja uma relação de longo prazo, pois dificuldades 
importantes aparecem para o contratante em uma eventual troca de prestador de serviço.

Os serviços associados a bens, em muitos casos, têm sido uma expressiva fonte de receita e de lucro (GEBAUER; FLEISCH, 2007). Rese et al. (2009) citam como exemplo a indústria automotiva em que cerca de $60 \%$ da receita é gerada depois que o veículo foi vendido. Viardort (2004) menciona a indústria aeronáutica, na qual o serviço prestado após a venda pode representar $80 \%$ da receita. Wagner et al. (2007) citam que, em fabricação e venda de tratores, até um terço do resultado pode vir de operações que ocorreram após a venda. Bundschuh e Dezvane (2003) apontam que operações de pós-venda podem faturar até cinco vezes o preço do produto ao longo do ciclo de vida. Em alguns casos, o produtor de bens com serviços associados assume os riscos e a total responsabilidade pela operação do sistema no cliente, o que significa, além de receita adicional, agregação de valor (GEBAUER, 2008).

Relacionado ao cenário descrito, observa-se a presença recente de termos como servitização e product-service system ou sistemas produto-serviço (PSS) na literatura relativa à gestão de operações. Como exemplos citam-se: Rese et al. (2009a) que estudaram os elementos preferenciais dos consumidores presentes na oferta do PSS; Sakao et al. (2009) que discutem as diversas abordagens do PSS; Kimita et al. (2009) que propõem um método para avaliar a satisfação dos consumidores de PSS; as diversas configurações de PSS são apresentadas em Aurich et al. (2009) e em Tukker (2004); Azarenko et al. (2009) discutem as implicações do PSS para a indústria de máquinas ferramentas; Vezzoli e Sciama (2008) discutem casos de aplicação de PSS em países emergentes; Spring e Araújo (2009) propõem um repensar das estratégias das operações de PSS.

Apesar de relevante, estudos sobre PSS são recentes e, até onde se pesquisou, não se observou material organizado que apresentasse um quadro de trabalho sobre as estruturas de um PSS ou sobre os elementos relevantes no projeto e implantação do mesmo. Este trabalho tem por objetivo caracterizar o PSS, identificando suas diversas categorias e discutindo os elementos viabilizadores para a transição de empresa de manufatura para empresa de fornecedora de um sistema integrado de bens e serviços. Também é objetivo organizar esses conceitos e 
reflexões em um quadro de trabalho e apontar direções para futuras pesquisas no tema. Nesse contexto, entende-se que é possível avançar em direção a uma base conceitual que ofereça subsídios iniciais para ulteriores pesquisas.

\section{MÉTODO DE PESQUISA}

A presente pesquisa pode ser considerada exploratória pois objetiva contribuir para a formação de uma base de conhecimento destinada a suportar futuras pesquisas empíricas, aplicadas em gestão de operações de sistemas produto-serviço (PSS).

Quanto ao tipo, é bibliográfica; foi assumida uma perspectiva crítica de análise. Pretende-se aumentar o entendimento sobre os elementos essenciais a serem considerados na concepção de um PSS, revisando abordagens presentes na literatura. Fontes secundárias foram a base da pesquisa bibliográfica para a formação do marco referencial teórico. A pesquisa teórica não implica em imediata intervenção na realidade, mas nem por isso deixa de ser importante, pois seu papel é decisivo na criação de condições para a intervenção (SALVADOR, 1981).

Este estudo se divide em dois estágios: o primeiro trás o resultado de um levantamento bibliográfico sobre PSS e temas afins tais como servitização; o segundo, partindo da análise do levantamento bibliográfico, apresenta propostas de pesquisa em PSS.

Assim como em Almeida et al. (2008) e em Baines et al., (2007), o levantamento bibliográfico apoiou-se em consultas a periódicos disponibilizados em bases de dados tais como Emeraldinsight, Blackwell-synergy, Sciencedirect e CAPES. Inicialmente, foram buscados artigos em que o termo "product-service system" comparecia no título ou em palavras-chave. Na seqüência, o termo "servitização" foi pesquisado por ser considerado complementar ao PSS (ALMEIDA et al., 2008). Por fim, foram identificadas outras referências em que o objeto de estudo era a transição da manufatura para oferta de serviços ou soluções.

A proposição de pesquisas futuras, a partir de um quadro de trabalho, foi construída analisando-se o levantamento bibliográfico efetuado. 


\section{LEVANTAMENTO BIBLIOGRÁFICO}

O Quadro 1 apresenta o resultado da pesquisa bibliográfica; artigos relacionados com prodcut-service system, com servitização e cujo objeto de estudo era a transição da manufatura para oferta de serviços ou soluções foram consultados. Buscou-se a identificação das temáticas relacionadas ao PSS que vêm sendo pesquisadas e publicadas. 


\begin{tabular}{|c|c|c|c|c|c|c|c|}
\hline \multirow[b]{2}{*}{ Referência } & \multirow[b]{2}{*}{ Foco } & \multirow[b]{2}{*}{ Principais achados } & \multicolumn{5}{|c|}{ enfoque } \\
\hline & & & 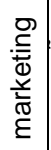 & 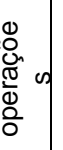 & 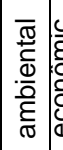 & 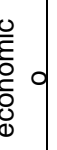 & 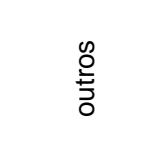 \\
\hline Rese, M. et al. (2009b) & $\begin{array}{l}\text { Alternativas de PSS baseadas } \\
\text { nas preferências dos } \\
\text { consumidores }\end{array}$ & $\begin{array}{l}\text { Apresentar drivers relacionados aos consumidores para } \\
\text { diferentes tipos de PSS }\end{array}$ & $\mathrm{x}$ & $\mathrm{x}$ & & & \\
\hline Sakao, T. et al. (2009) & $\begin{array}{l}\text { Propor um framework para as } \\
\text { diversas abordagens de PSS }\end{array}$ & Considerar potencial de mercado e questão ambiental & $\mathrm{x}$ & & $\mathrm{x}$ & & \\
\hline Kimita, K. et al. (2009) & $\begin{array}{l}\text { Satisfação do consumidor em } \\
\text { relação ao PSS }\end{array}$ & Rever as formas de avaliação da satisfação do consumidor & $\mathrm{x}$ & $\mathrm{x}$ & & & $\begin{array}{l}\text { qualidade; } \\
\text { satisfação }\end{array}$ \\
\hline Aurich, J. et al (2009) & Configuração de PSS & $\begin{array}{l}\text { Expandir a visão de ciclo de vida do produto para ciclo de } \\
\text { vida do PSS }\end{array}$ & & $\mathrm{x}$ & $\mathrm{x}$ & & Satisfação \\
\hline Azarenko, A. et. al (2009) & $\begin{array}{l}\text { Desenvolvimento de PSS para } \\
\text { produtos de base tecnológica }\end{array}$ & $\begin{array}{l}\text { Mudança na estrutura tradicional de desenvolvimento de } \\
\text { produtos }\end{array}$ & & $\mathrm{x}$ & & & projeto \\
\hline Vezzoli, C.; Sciama, D. (2007) & $\begin{array}{l}\text { Aplicação e desenvolvimento de } \\
\text { PSS em países emergentes }\end{array}$ & Relatos com foco educacional & & & & & ensino \\
\hline Morelli, N. (2003) & $\begin{array}{l}\text { O PSS sob a perspectiva dos } \\
\text { designers }\end{array}$ & $\begin{array}{l}\text { Necessidade de mudança de abordagem na concepção dos } \\
\text { produtos / serviços / PSS }\end{array}$ & & $\mathrm{x}$ & $\mathrm{x}$ & & projeto \\
\hline Spring, M.; Araújo, L. (2009) & $\begin{array}{l}\text { Mudanças na forma de } \\
\text { consumo introduzidas pelo PSS }\end{array}$ & $\begin{array}{l}\text { Mudanças na forma de consumo implicam em mudanças na } \\
\text { forma de produzir e comercializar }\end{array}$ & $x$ & $\mathrm{x}$ & $\mathrm{x}$ & & \\
\hline Tukker, A. (2004) & Categorização dos tipos de PSS & Características das categorias de PSS & & $\mathrm{x}$ & $\mathrm{x}$ & $\mathrm{x}$ & \\
\hline Rese, M. et al. (2009a) & $\begin{array}{l}\text { Projeto de PSS orientado ao } \\
\text { produto }\end{array}$ & $\begin{array}{l}\text { Mudança no processo de desenvolvimento e concepção de } \\
\text { produtos }\end{array}$ & & $\mathrm{x}$ & & & projeto \\
\hline Yang, X. et al. (2009) & $\begin{array}{l}\text { Método para desenvolvimento } \\
\text { do PSS considerando ciclo de } \\
\text { vida ampliado }\end{array}$ & $\begin{array}{l}\text { Análise do ciclo de vida de produto passa a ser vista como } \\
\text { análise do ciclo de vida do PSS }\end{array}$ & & $\mathrm{x}$ & $\mathrm{x}$ & & \\
\hline Mont, O. (2002) & $\begin{array}{l}\text { Ganhos ambientais decorrentes } \\
\text { do PSS }\end{array}$ & $\begin{array}{l}\text { Discute e propõe investigar os efetivos ganhos ambientais do } \\
\text { PSS }\end{array}$ & & & $\mathrm{x}$ & & \\
\hline Aurich, J. et al. (2006) & Ciclo de vida do PSS & $\begin{array}{l}\text { Proposta de modularização de produtos da base tecnológica } \\
\text { para aumentar ganhos ambientais }\end{array}$ & & $\mathrm{x}$ & $\mathrm{x}$ & & \\
\hline Kang, M.; Wimmer, R. (2009) & $\begin{array}{l}\text { PSS como um novo modelo de } \\
\text { consumo }\end{array}$ & $\begin{array}{l}\text { Novo modelo de consumo conduz a novos modelos de } \\
\text { produção e comercailização }\end{array}$ & $\mathrm{x}$ & $\mathrm{x}$ & $\mathrm{x}$ & $\mathrm{x}$ & \\
\hline Cook, M. et al. (2006) & Casos de aplicação de PSS no & Apresenta casos de aplicação de PSS idealizados na & $\mathrm{x}$ & $\mathrm{x}$ & & $x$ & \\
\hline
\end{tabular}

Revista Produção Online, v.10, n.4, p. 837-860, dez., 2010 


\begin{tabular}{|c|c|c|c|c|c|c|c|}
\hline \multirow[b]{2}{*}{ Referência } & \multirow[b]{2}{*}{ Foco } & \multirow[b]{2}{*}{ Principais achados } & \multicolumn{5}{|c|}{ enfoque } \\
\hline & & & 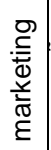 & 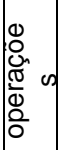 & 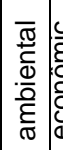 & 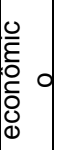 & 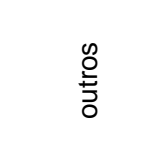 \\
\hline & Reino Unido & Universidade e, posteriormente, adotados por empresas & & & & & \\
\hline Willians, A. (2006) & PSS na indústria automotiva & Caso de aplicação do PSS & & $x$ & & & \\
\hline Mont, O.; Lindqvist, T, (2003) & $\begin{array}{l}\text { Políticas públicas para apoiar o } \\
\text { desenvolvimento de PSS }\end{array}$ & Apresenta propostas e casos & $x$ & & $x$ & $x$ & \\
\hline Tukker, A.; Tischner, U. (2006) & PSS: passado, presente e futuro & $\begin{array}{l}\text { Discute tendências e a mudança nos hábitos de consumo e } \\
\text { produção }\end{array}$ & $\mathrm{x}$ & $\mathrm{x}$ & $\mathrm{x}$ & $\mathrm{x}$ & \\
\hline Krucken, L.; Meroni, A. (2006) & $\begin{array}{l}\text { Experiências práticas de } \\
\text { desenvolvimento de PSS }\end{array}$ & Papel da comunicação na comercialização do PSS & $x$ & & & & \\
\hline Manzini, E.; Vezolli, C. (2003) & $\begin{array}{l}\text { Design estratégico buscando } \\
\text { sustentabilidade ambiental }\end{array}$ & Discute o papel do PSS na redução do impacto ambiental & & & $x$ & & \\
\hline An, Y. et al. (2008) & $\begin{array}{l}\text { Desenvolvimento integrado de } \\
\text { PSS }\end{array}$ & $\begin{array}{l}\text { Propõe uso de QFD (Desdobramento da Função Qualidade) } \\
\text { no desenvolvimento de PSS }\end{array}$ & & $\mathrm{x}$ & $\mathrm{x}$ & & qualidade \\
\hline Roy, R. (2000) & Sistemas de PSS sustentáveis & $\begin{array}{l}\text { Entender o que vem a ser sustentabilidade ambiental de um } \\
\text { PSS }\end{array}$ & & & $\mathrm{x}$ & & \\
\hline Baines, T. et al. (2007) & Servitização e PSS & Barreiras e vantagens & $\mathrm{x}$ & $\mathrm{x}$ & $\mathrm{x}$ & $\mathrm{x}$ & \\
\hline Baines, T. et al. (2009) & Servitização da manufatura & $\begin{array}{l}\text { Trás elementos para pesquisa futura sobre o impacto nas } \\
\text { operações decorrentes da servitização }\end{array}$ & & $\mathrm{x}$ & & & \\
\hline Pawar, K. et al. (2009) & $\begin{array}{l}\text { PSS como elemento de criação } \\
\text { de valor }\end{array}$ & $\begin{array}{l}\text { Discute as relações entre projeto do produto, do serviço e } \\
\text { aspectos organizacionais para a criação de valor através do } \\
\text { PSS }\end{array}$ & $\mathrm{x}$ & $x$ & & $\mathrm{x}$ & \\
\hline Johnstone, S. et al. (2009) & $\begin{array}{l}\text { Caso de aplicação do PSS no } \\
\text { setor aeroespacial }\end{array}$ & $\begin{array}{l}\text { Apresenta as fases de desenvolvimento e implantação do } \\
\text { PSS na indústria aeroespacial }\end{array}$ & & $x$ & & & \\
\hline Brax, S.; Jonsson, K. (2009) & $\begin{array}{l}\text { Proposta de solução integrada } \\
\text { em serviços de manutenção }\end{array}$ & Apresenta dois casos comparativos aplicados na manufatura & & $\mathrm{x}$ & & & \\
\hline Almeida, L. et al. (2008) & $\begin{array}{l}\text { Revisão da literatura sobre } \\
\text { servitização e PSS }\end{array}$ & $\begin{array}{l}\text { Número de estudos em servitização e PSS é limitado e } \\
\text { recente }\end{array}$ & $x$ & $\mathrm{x}$ & & $\mathrm{x}$ & \\
\hline
\end{tabular}

Quadro 1 - Levantamento bibliográfico sobre PSS, servitização e temas afins 
A análise decorrente do levantamento bibliográfico possibilitou: o estudo do PSS sob o enfoque ambiental e sob o enfoque econômico, a apresentação das categorias de PSS e suas respectivas características; a compreensão dos elementos presentes na configuração e desenvolvimento de um PSS, incluindo análise do ciclo de vida do PSS; a discussão sobre elementos vinculados a qualidade do PSS; e a identificação de barreiras e vantagens do PSS.

\subsection{CONTEXTUALIZAÇÃO DO PRODUCT-SERVICE SYSTEM (PSS)}

O conceito de PSS teve sua origem, nos anos 1990, no norte da Europa. Iniciou com uma abordagem focada na ecologia industrial, centrada no conceito de "vender performance" em vez de vender "bens" (SPRING; ARAÚJO, 2009). PSS é um caso de servitização e pode ser concebido como uma extensão da operacionalidade de um bem físico, agregando a esse serviços especializados. $O$ que é vendido é o direito de uso do bem, não o direito de propriedade. Mesmo em uso pelo cliente, o bem ainda pertence ao fabricante. Portanto, o cliente pode permanentemente reorganizar sua estrutura produtiva, trocando facilmente de ativos segundo evolua sua estratégia de manufatura. Já o fabricante pode ter acesso permanente a informações de uso do produto que podem permitir melhorias no projeto, com aumento de desempenho e confiabilidade (BAINES et al, 2007). Tais melhorias surgem pela mensuração e entendimento de fenômenos que só se manifestam ao longo do ciclo de vida do equipamento e seriam inacessíveis aos ensaios usuais que fabricantes fazem durante a fabricação, montagem e posta-emmarcha de equipamentos complexos. Como exemplo, citam-se os desgastes por uso que marcam a terceira fase da curva da banheira (mortalidade senil, ou wear-out); a menos que se executem ensaios acelerados que ofereçam estimativas para estas incidências, esses desgastes não serão detectados em fábrica ou em tempo de posta-em-marcha; apenas o serão durante o ciclo de vida do equipamento.

Baines et al. (2007) citam o caso da Rolls-Roice PLC., fabricante de turbinas a gás para aviação, que não vende suas turbinas para as companhias aéreas, mas potência por hora. $O$ acesso permanente aos equipamentos permite que o fabricante aprimore parâmetros de desempenho da turbina e também de outros elementos do 
sistema de propulsão, tal como motores e demais componentes. Pode-se acrescentar o regime de entregas consignadas de máquinas copiadoras que a Xerox adotou nos anos 1970. A principal motivação da empresa, na ocasião, era mercadológica. Dada a diferença de tecnologia existente na época, em relação aos concorrentes, a empresa entendia ser mais vantajoso alugar ou compartilhar máquinas e não vendê-las aos usuários. Na ocasião, uma importante fonte de receita da empresa era o serviço de treinamento e assistência técnica.

Dentro do contexto ambiental, Manzini e Vezzoli (2003) definem PSS como sendo uma estratégia de inovação em que o foco do negócio de uma empresa migra do projeto (e comercialização) de produtos físicos para projeto (e comercialização) de um sistema de bens e serviços que atuam de forma conjunta para satisfazerem uma demanda específica do cliente. Baines et al. (2009) e Roy (2000) ressaltam que, até o presente, a maior parte das publicações sobre PSS ainda estão centradas nas questões ambientais e podem ser encontradas em periódicos relativos à sustentabilidade e produção mais limpa. Como exemplos desses artigos citam-se: Manzini e Vezolli (2003), Kang e Wimmer (2009), Roy (2000), Morelli (2003), Aurich et al. (2009) e Sakao et al. (2009).

Para Manzini e Vezzoli (2003), a mudança de enfoque de produtos físicos para um sistema integrado de bens e serviços visa oferecer soluções para os consumidores. Em termos ambientais, uma das possíveis contribuições do PSS é a maximização do uso dos recursos, visto que em muitos casos não será mais necessário que cada consumidor adquira o produto físico mas, sim, adquira o uso do bem físico, associado a um serviço, apenas nos períodos em que o mesmo é demandado. Exemplos de PSS podem ser vistos em Besch (2003), em Mont e Lindqvist (2003) e em Johnstone et al. (2009).

McAloone e Andreasen (2004) consideram relevante identificar em um PSS as seguintes características: (i) no tempo, as fases ao longo do ciclo de vida; (ii) no âmbito do artefato, os múltiplos e interrelacionados sub-sistemas, tanto físicos como gerenciais (manutenção, reformas, reposições, desempenho); (iii) quanto ao valor, os diversos interessados na utilização e na geração de benefícios e ônus; e (iv) no campo social, identificar atitudes e responsabilidades com o sistema ambiental resultante. 
Contrapondo o exposto, Sakao et al. (2009) argumentam que o PSS pode levar a uma acelerada reposição dos produtos, visto que muitos usuários não propiciarão aos produtos os mesmos cuidados caso esses fossem de sua propriedade. O produto pode estar sendo exposto a um uso mais intensivo para o qual não foi projetado. Somente melhorias no desempenho ambiental dos produtos e no desempenho do projeto podem, respectivamente, contribuir para reduzir o impacto ambiental ou aperfeiçoar o uso dos produtos.

Em síntese, as questões que vêm sendo pesquisadas dentro do contexto ambiental do PSS se referem à mensuração dos benefícios ambientais e ao projeto do PSS, incluindo remanufatura, uso de produtos e componentes (MONT, 2002).

A segunda abordagem dada ao PSS é motivada por aspectos econômicos e mercadológicos. Nesse sentido, para Kimita et al. (2009), o PSS é um conceito em que serviços são adicionalmente oferecidos com produtos físicos para aumentar o valor do pacote ofertado ao cliente. O projeto do PSS tem importante papel, pois o serviço deve ser projetado para toda a cadeia de atividades, incluindo a política relativa à gestão das pessoas; já os produtos físicos devem ser projetados para satisfazer os consumidores com relação ao seu desempenho.

É possível compreender o papel dos serviços de alto valor agregado observando a evolução das organizações. Para Pawar et al. (2009), no início da produção em massa, o produto físico era visto como o elemento que agregava valor e serviços adicionais eram percebidos como agregadores de custos para o produtor; posteriormente, agregar serviços aos produtos físicos passou a ser elemento de diferenciação; por fim, no PSS, a combinação de produto físico com serviços capazes de oferecer soluções aos consumidores, é vista de forma integrada como gerador de valor e diferenciação perante clientes. A Figura 1 ilustra a evolução das empresas, que passaram de produtoras de bens ou serviços para produtoras de bens agregados de serviços e, por fim, para produtoras de valor por meio do PSS. 


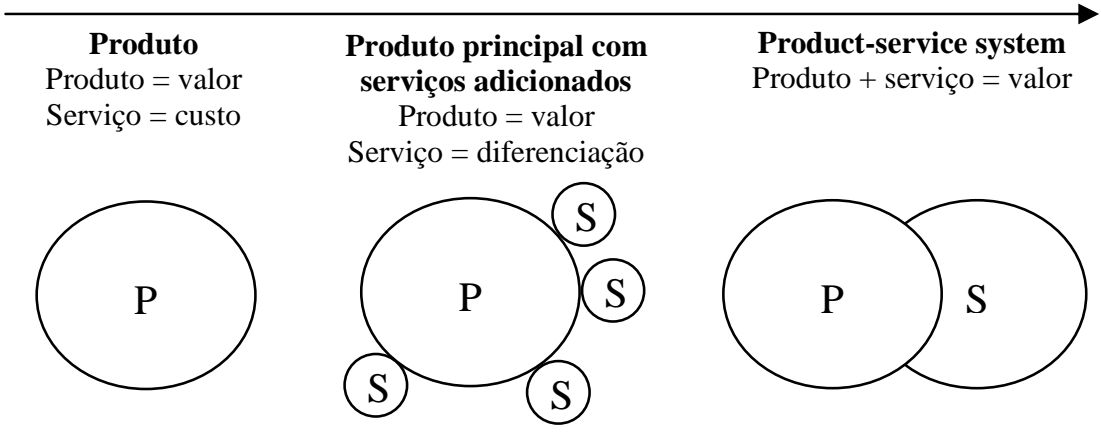

Figura 1- Evolução de produção de produto ou serviço para product-service system Fonte: Adaptado de Pawar et al., (2009)

O PSS pode contribuir com a sustentabilidade ambiental, pelos motivos já apresentados; em um segundo momento, pode melhorar a sustentabilidade financeira da organização através da geração de receitas provenientes de uma relação de longo prazo com o consumidor; e pode, ao evoluir, levar a co-criação de valor, através do envolvimento de vários parceiros gerando ao consumidor uma experiência diferenciada (PAWAR et al., 2009). O Quadro 2 ilustra a evolução do PSS considerando a transição do enfoque ambiental para o financeiro e, por fim, para a co-criação de valor.

\begin{tabular}{|c|c|c|c|}
\hline & $\begin{array}{l}\text { Fase um: início do } \\
\text { product-service } \\
\text { system }\end{array}$ & $\begin{array}{l}\text { Fase dois: PSS com } \\
\text { soluções integradas }\end{array}$ & $\begin{array}{c}\text { Fase três: PSS com } \\
\text { serviços } \\
\text { "experienciais" }\end{array}$ \\
\hline Objetivo da transição & $\begin{array}{c}\text { Sustentabilidade } \\
\text { ambiental }\end{array}$ & $\begin{array}{l}\text { Sustentabilidade } \\
\text { financeira }\end{array}$ & Co-criação de valor \\
\hline $\begin{array}{l}\text { Objetivos a serem } \\
\text { atingidos }\end{array}$ & $\begin{array}{l}\text { Combinar produtos e } \\
\text { serviços para prover } \\
\text { valor derivado dos } \\
\text { produtos físicos e } \\
\text { gerando redução do } \\
\text { impacto ambiental. }\end{array}$ & $\begin{array}{l}\text { Adicionar serviços } \\
\text { aos produtos físicos } \\
\text { existentes, provendo } \\
\text { valor aos } \\
\text { consumidores em } \\
\text { relações de longo } \\
\text { prazo o que trará } \\
\text { receitas no longo } \\
\text { prazo. }\end{array}$ & $\begin{array}{l}\text { Criar um ambiente no } \\
\text { qual serviços e } \\
\text { produtos físicos } \\
\text { geram experiências } \\
\text { diferenciadas, } \\
\text { levando à fidelidade. }\end{array}$ \\
\hline
\end{tabular}

Quadro 2- Evolução do PSS

Fonte: Adaptado de Pawar et al. (2009)

A implementação do PSS é complexa; prover soluções envolve diversas possibilidades e os serviços são dinâmicos, apresentando uma parcela significativa de intangibilidade. O PSS gera uma mudança na forma de consumir o produto, ampliando o uso de leasing, pagamento pelo uso ou ainda pagamento vinculado ao 
resultado obtido. A mudança da abordagem tradicional de comercialização de bens e/ou serviços para a comercialização de PSS pode gerar maiores custos fixos aos produtores e enfatizar os custos variáveis para os consumidores (SPRING; ARAÚJO, 2009).

O desenvolvimento de um PSS precisa considerar o envolvimento do consumidor, fortalecendo as relações do produtor com o mesmo em todas as fases do ciclo de vida do PSS. Desenvolver níveis de ofertas que transcendem as barreiras organizacionais entre consumidores e produtores requer o alinhamento entre a estratégia e as operações e requer o desenvolvimento conjunto de produto. Necessita-se, ainda, desdobrar as oportunidades de negócio para, após a agregação de serviços aos produtos físicos, conseguir migrar para a oferta de soluções (SAKAO et al., 2009).

\subsection{CATEGORIAS DE PRODUCT-SERVICE SYSTEM}

Autores como Azarenko et al. (2009), Rese et al. (2009a) e Yang et al. (2009) apresentam três categorias de PSS: (i) product-oriented service; (ii) useoriented service e (iii) result-oriented service. Cada uma dessas categorias tem particularidades com relação aos aspectos econômicos e ambientais. Tukker (2004) desdobrou as mesmas, apresentando oito tipos de PSS a seguir descritos.

- Product-oriented service

a) Product-related service - o produtor vende um produto físico e oferece serviços que são necessários durante a fase de uso do produto (exemplo: contrato de manutenção).

b) Assessoria e consultoria - com relação ao produto físico vendido, o produtor ou provedor de serviços fornece assessoria para a obtenção de uso mais eficiente (exemplo: otimizar a logística de uma fábrica onde os produtos são produzidos em lotes unitários; prover treinamento organizacional para o uso do produto).

- Use-oriented service. 
a) Leasing de produtos - um provedor de serviços mantém o direito de propriedade do bem e tem a responsabilidade em definir as condições de uso do mesmo, sendo, muitas vezes, responsável pela manutenção, reparo, controle. Nesse caso, há o pagamento regular de uma taxa para o uso.

b) Aluguel de produtos ou compartilhamento - os produtos, em geral, são de propriedade de um provedor de serviços que tem a responsabilidade pela manutenção, reparo e controle. O usuário não tem acesso ilimitado e individual ao produto físico; o mesmo produto pode ser utilizado seqüencialmente por diferentes usuários.

c) Product pooling - similar ao aluguel de produtos ou compartilhamento, porém há um simultâneo uso do produto físico por diversos usuários.

- Result-oriented service.

a) Atividade de gerenciamento e terceirização - parte das atividades de uma empresa é terceirizada para outras empresas. Como os terceiros são medidos através de indicadores de desempenho, esse tipo de PSS está incluído em result-oriented service.

b) Pagamento por unidade de serviço - esse tipo de PSS ainda tem como base um produto físico, mas o usuário não compra o produto físico e sim um resultado desse produto de acordo com o nível de serviço (exemplo: pagamento de cópias impressas ao provedor de serviços de impressão, proprietário das impressoras).

c) Resultados funcionais - o provedor de serviços acorda com o cliente o resultado a ser entregue (exemplo: oferecer ambiente termicamente agradável no lugar de comercializar o equipamento de ar condicionado).

\subsection{O PROJETO DE UM PRODUCT-SERVICE SYSTEM}

Analisando-se os tipos de PSS descritos, nota-se a presença de diversos atores. Esse aspecto foi abordado por Morelli (2003). Um PSS pode ser visto como uma "construção social" baseada em forças de atração tais como objetivos, resultados esperados e critérios para a solução de problemas que podem congregar diversos atores em relações de parcerias de longo prazo. Um PSS é o resultado de 
um processo de co-produção de valor envolvendo tais parceiros. Sua efetividade é baseada na visão compartilhada e na análise dos possíveis cenários a serem considerados.

Ehrenfeld (2001) relaciona o PSS com o ecodesign ao examiná-lo, criticamente, como sustentabilidade expandida no tempo. $O$ autor também considera aspectos sociais e humanísticos, principalmente aqueles ligados aos sentimentos de consumismo crescente e de posse, que podem sabotar esforços de desenvolvimento de PSS se forem negligenciados.

$\mathrm{Na}$ discussão que fundamenta iniciativas em prol da sustentabilidade, tais como ecodesign e PSS, existe convergência no que se refere à necessidade de aumentar o desempenho ambiental de produtos e serviços, caso se queira obter sustentabilidade ecológica (REIJNDERS, 1998). O autor criou o fator $x$, segundo o qual o desempenho ambiental de um sistema produtivo deve aumentar $x$ vezes $(2,4$, 10 ou 20), mesmo que se desconheça esse valor. Segundo McAloone e Andreasen (2004), independentemente do valor de x, é necessário mudar o foco dos esforços de projeto de simples artefatos inovativos para inteiros sistemas produto-serviço, incluindo os aspectos sociotécnicos e comportamentais. A relação usual entre fabricante-revendedor e usuário pode ser rearranjada para que se obtenham benefícios econômicos e ambientais para todas as partes.

A atividade de projeto de um PSS deve ser vista, portanto, como um elemento catalizador capaz de gerar coesão entre os parceiros. Isso significa, para Morelli (2003), que os projetistas devem ter ferramentas para:

- trabalhar na identificação dos atores presentes no PSS, definidos a partir de uma análise analítica;

- trabalhar com diversos cenários de PSS verificando casos de uso, seqüências de ações e o papel de cada ator; devem, também, definir os requerimentos para o PSS e a estrutura lógica e organizacional necessária; e,

- trabalhar com ferramentas de gerenciamento que representem o PSS como um todo, bem como todos os seus componentes, isto é os elementos físicos, os logísticos e as seqüências temporais. 
Ao efetuar a análise dos diversos cenários de PSS, a empresa deve compreender seu atual posicionamento: (i) produtora de bens físicos puros; (ii) produtora de bens tangíveis com serviços associados; (iii) produtora de bens físicos e de serviços (híbrida); (iv) provedora de serviços com acompanhamento de bens físicos; ou (v) provedora ou produtora de serviços puros. Posteriormente, cabe uma análise da possibilidade de avançar em direção ao PSS considerando: (i) mercado; (ii) parceiros; (iii) infraestrutura; e (iv) aspectos culturais (MORELLI, 2003).

Pawar et al. (2009) observam que o projeto de um PSS inicia com a definição da proposição de valor que irá satisfazer os consumidores, devendo a mesma ser claramente definida; os custos-alvo precisam ser estimados, bem como os riscos envolvidos. Posteriormente, parte-se para projetar o PSS que irá criar o valor definido; deve-se, então, projetar a organização em termos da capacidade requerida para complementar as competências essências da empresa. Por fim, fazse necessário identificar, selecionar e gerenciar a rede de parceiros que juntamente com a organização irão entregar valor, ou seja, o PSS. A construção da rede de parceiros, ainda na fase de projeto do PSS, deve considerar também como se dará a comunicação, para cada ator, sobre o que esperar desse PSS (KRUCKEN e MERONI, 2006). Tukker e Tischner (2006) incluem a abordagem ambiental. Nesse contexto, o projetista de um PSS precisa: (i) focar nas funcionalidades finais ou satisfação do usuário e (ii) elaborar um sistema de negócio que provê essas funcionalidades com sustentabilidade ambiental. A Figura 2 ilustra as fases do projeto de um PSS. 


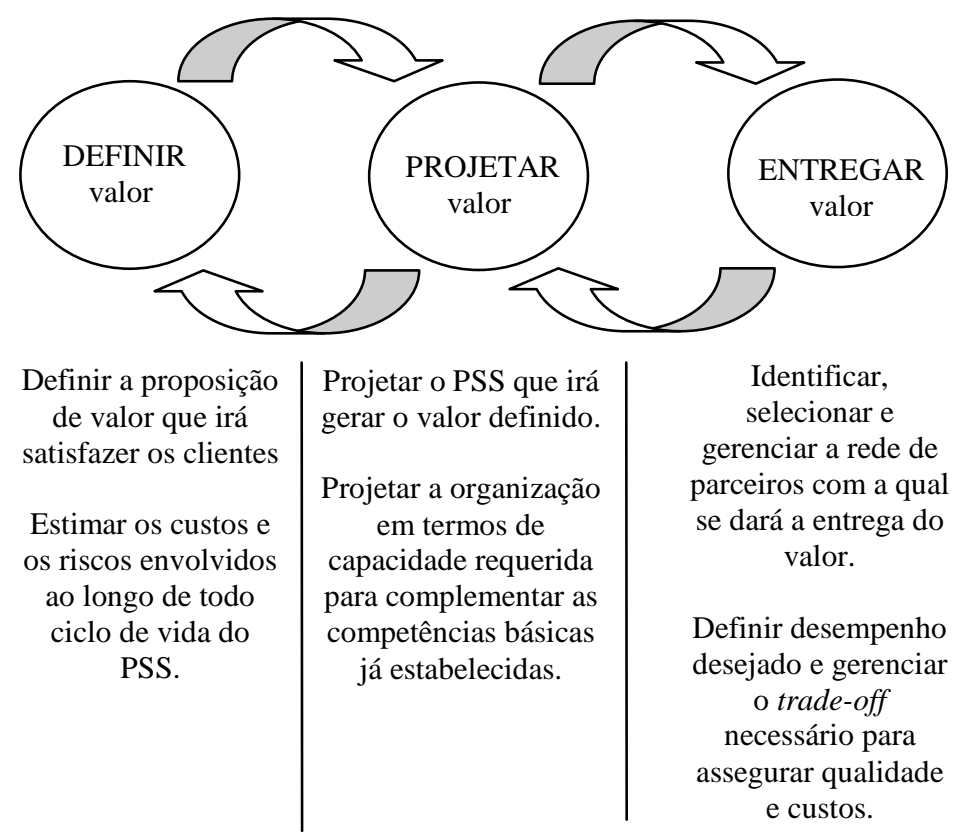

Figura 2 - Fases do projeto de um PSS

Fonte: Adaptado de Krucken e Meroni (2006)

Johnstone et al. (2009) citam três fases observadas na transição de uma empresa tipicamente de manufatura para PSS. As mesmas devem ser consideradas no projeto do PSS. Na primeira fase, produtos e serviços são combinados e oferecidos de forma integrada. Na segunda, o portfólio de produtos é ampliado através da agregação de outros serviços adicionais. Por fim, são ofertadas diversas combinações de produtos físicos e serviços visando a entrega de soluções.

Em termos de ferramentas e técnicas a serem utilizadas no projeto de um PSS, An et al. (2008) sugerem o uso do QFD (Desdobramento da Função Qualidade). O mesmo deve considerar o produto de modo amplo, incluindo os aspectos tangíveis (produto físico) e intangíveis (serviços).

\subsection{QUALIDADE EM PSS}

Pode-se pensar na qualidade sob o ponto de vista das operações, o que remete para o planejamento da qualidade, a garantia da qualidade a ser obtida através do projeto dos produtos e processos, o controle da qualidade e a melhoria da qualidade (AN et al., 2008). 
Behara e Gundersen (2001), ao compararem empiricamente os aspectos presentes na gestão da qualidade em manufatura com gestão da qualidade em serviços, observaram que as distinções entre os mesmos são muitas. Sob o ponto de vista de um PSS, manufatura e serviço andam juntos. Cabe, nesse contexto, o estabelecimento de um sistema de gestão da qualidade ampliado, capaz de contemplar ambos.

Sob a perspectiva do consumidor, o resultado da entrega de um produto (bem ou serviço ou ambos), irá gerar um nível de satisfação. Esse, por sua vez, conduzirá a relações de longo prazo que terão como efeito maior lucratividade para a organização (RANAWEERA; PRABHU, 2003).

Diversas dimensões da qualidade podem estar presentes em um produto físico ou serviço ou combinação de ambos. No entanto, Ramos et al. (2009) identificaram diversas abordagens para as dimensões da qualidade, ilustradas no Quadro 3. O modelo das lacunas da qualidade proposto por Parasunaman et al. (1985) baseia-se nas dimensões da qualidade presentes em um servi;o.

Por outro lado, até onde se pesquisou, não foram encontradas publicações que tratem especificamente da questão da qualidade em PSS, seja sob o ponto de vista das operações seja sob a ótica do consumidor.

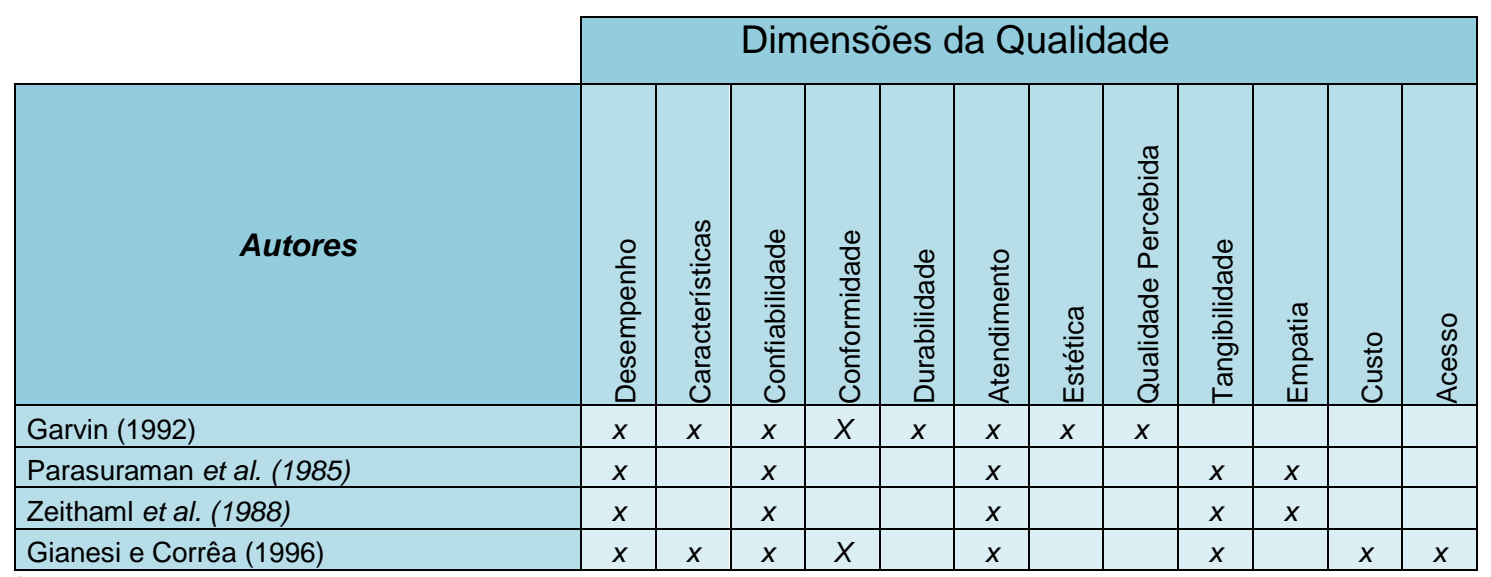

Quadro 3 - Dimensões da qualidade

Fonte: Ramos et al. (2009) 


\subsection{ANÁLISE DE UM PRODUCT-SERVICE SYSTEM}

Nem toda organização deseja ou pode combinar serviços com produtos; ou ainda, nem toda organização pode ser capaz de fazê-lo. Nem todas as iniciativas de PSS são aceitas pela rede de parceiros ou pelos consumidores. Aspectos econômicos e ambientais são relevantes, mas identificar o estilo de liderança, a estrutura da organização, as habilidades e competências da força de trabalho e da rede de parceiros podem essenciais para a elaboração de um PSS (MORELLI, 2003).

A análise de um PSS, para Morelli (2003), pode ser apoiada em três perspectivas, a saber: (i) sob o ponto de vista do marketing de produto: nesse caso, a noção de PSS deriva da evolução da idéia de um produto físico para uma entidade; isso reduz a ênfase nos materiais físicos levando a um produto cujo componente material é inseparável do imaterial, o sistema de serviços que será usado; (ii) sob o ponto de vista do marketing de serviços: o PSS representa a evolução da abordagem tradicional e padronizada para uma focada e personalizada; essa perspectiva impacta na lógica de produção em massa, presente em diversos setores da economia; e (iii) sob o ponto de vista do gerenciamento de produtos: a noção de PSS implica um novo jeito de usar o produto; tal jeito implica no modo como o mesmo deve ser concebido e projetado. A inserção de serviços em ambientes tradicionalmente de manufatura trará aspectos relacionados a intangibilidade dos serviços, heterogeneidade, inseparabilidade e perecibilidade.

As vantagens de um PSS, sintetizadas por Tukker (2004), são: (i) oferecer aos clientes soluções integradas e customizadas de modo que os mesmos possam ater-se as suas atividades principais; (ii) construir uma relação de longo prazo com os clientes, favorecendo a lealdade; (iii) propiciar mais velocidade na inovação visto que o foco é atender as necessidades dos clientes através de soluções; e (iv) diminuir o impacto ambiental dos produtos e os custos envolvidos em todo seu ciclo de vida.

Como barreiras para a adoção de um PSS observam-se: (i) os consumidores não enxergam benefícios; (ii) dificuldades no projeto de um PSS, decorrente do 
enfoque tradicional e formação dos projetistas; (iii) falta de aspectos regulatórios; e (iv) a cultura do "ter" (BAINES et al., 2009).

Goedkoop et al. (1999) propõem que a análise de um PSS se apóie em elementos ambientais, econômicos e internos. Inicialmente uma descrição precisa das funções a serem atendidas por um PSS deve ser efetuada. Nesse sentido, deve-se ter clareza de quais alternativas os clientes têm e qual o sistema de competição vigente no mercado em que a organização atua.

A abordagem qualitativa deve permitir, para a organização, a compreensão quatro aspectos:

i. Quais são as características ambientais necessárias durante 0 cumprimento da função do PSS e como estas se relacionam com o impacto global sobre a sociedade?

ii. Quais são as características econômicas no nível da organização e, também, em termos de setor e parceiros do negócio?

iii. De que forma o PSS se identifica com a identidade da organização e sua estratégia?

iv. Em que extensão o mercado aceitaria o PSS?

A análise do impacto ambiental de um PSS se inicia pela análise das fases do ciclo de vida em que o impacto ambiental dos produtos físicos é dominante. Como exemplos citam-se: casos em que o impacto de um PSS é dominante na fase de produção e descarte tais como embalagens; casos em que o impacto é dominante durante o uso tais como carros e televisores e casos em que o impacto é igualmente presente em todas as fases do ciclo de vida de um PSS como bens de consumo que necessitam cuidados constantes como roupas e bicicletas. A adição de serviços aos produtos físicos existentes poderá gerar um comportamento diferente com relação aos impactos ambientais uma vez que; (i) provê mobilidade extra para reparo ou entrega do serviço; (ii) prevê atualização das bases de serviços e dos hardwares e softwares instalados, sem necessariamente efetuar a reposição 
integral; e (iii) multiplica ou prolonga o uso dos produtos através do aluguel, compartilhamento ou outra forma de multiuso (GOEDKOOP et al., 1999).

A análise qualitativa econômica, ainda segundo os autores mencionados, deve incluir a análise do potencial de um PSS no mercado existente, considerando os custos e as receitas e a análise da criação de um novo mercado como resultado da adição de valor pelo PSS. As perspectivas do produtor e do consumidor devem ser avaliadas. O produtor deverá obter mais receitas através da otimização dos custos; devem ser considerados todos os custos e receitas para o produtor e para o consumidor durante todo o ciclo de vida do PSS.

Aspectos relativos à capacidade organização, recursos humanos e estruturais, estrutura de pós-venda para o PSS, capacidade de inovação da organização, parceiros envolvidos também precisam ser considerados.

\section{DISCUSSÃO E PROPOSTAS DE PESQUISAS FUTURAS}

A partir da análise da bibliografia sobre PSS utilizada para a elaboração deste trabalho, foi possível identificar algumas oportunidades de pesquisa a serem desenvolvidas, tanto em nível de pesquisa básica como em um contexto aplicado. A Figura 3 ilustra esquematicamente essas oportunidades. 


\section{CATEGORIAS DE PSS}
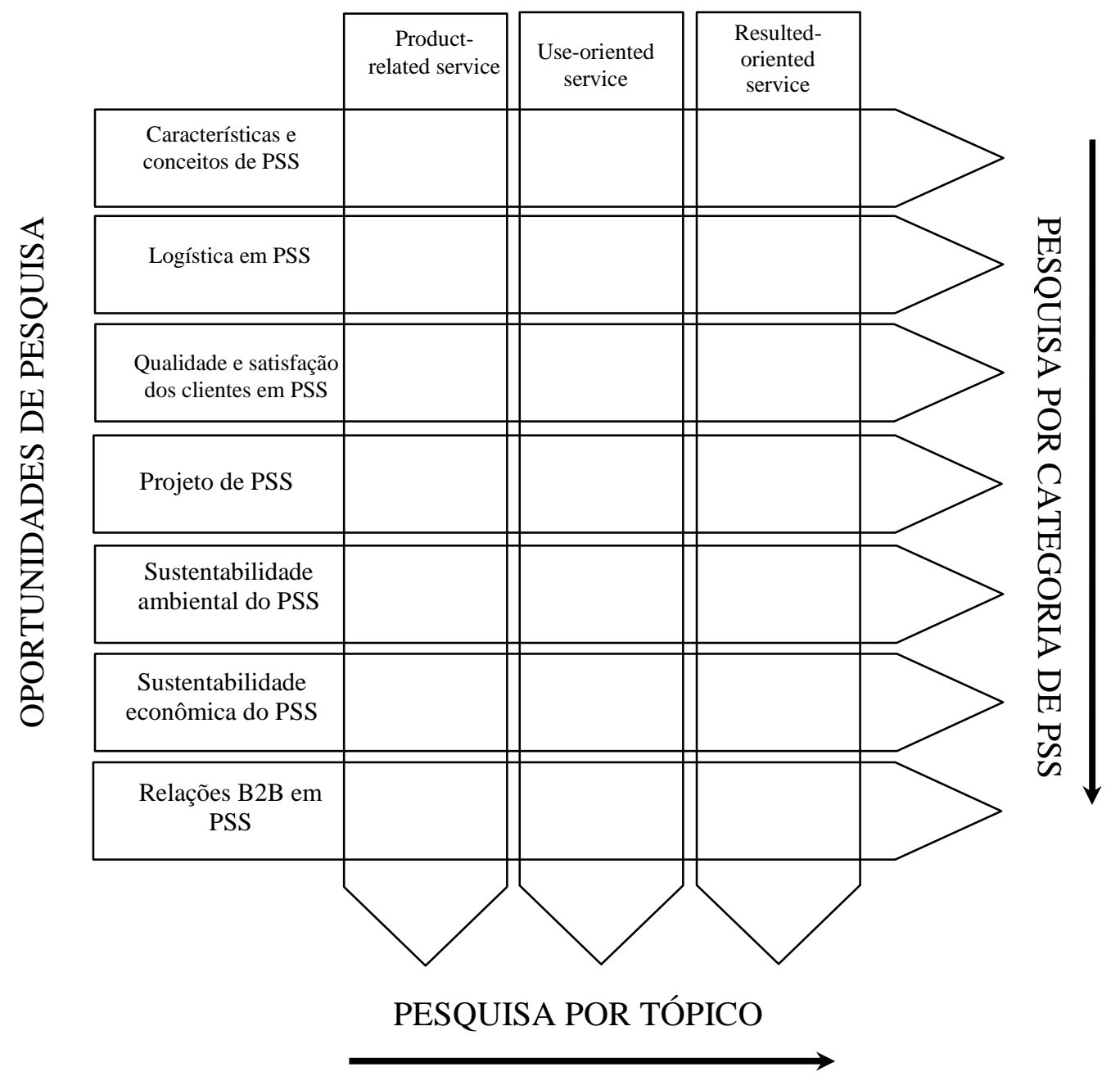

Figura 3 - Oportunidades de pesquisa por categorias de PSS

Nota-se que cada um dos cruzamentos dos eixos verticais e horizontais (células) pode ser visto como uma pesquisa potencial. Entende-se, também, como possibilidade a análise em profundidade de cada um dos tópicos indicados como "oportunidades de pesquisa" a serem executadas em cada categoria de PSS (eixos verticais). Cada "oportunidade de pesquisa" pode ainda ser realizada em estudos comparativos entre as categorias de PSS (eixos horizontais).

No que se refere às características das diversas categorias de PSS, julga-se relevante aprofundar as pesquisas identificando elementos estratégicos (Almeida et al., 2008), táticos e operacionais predominantes em cada categoria de PSS. Além da identificação dos elementos estratégicos, Johnstone et al. (2009) investigam as ferramentas de gerenciamento que representam um PSS em um caso de uma Revista Produção Online, v.10, n.4, p. 837-860, dez., 2010 
empresa da indústria aeroespacial. Partindo desse ponto, pode-se questionar acerca das ferramentas de gerenciamento nas diversas categorias de PSS.

Estabelecer a estratégia e o processo de entrega de um PSS, os respectivos canais, a definição das bases de serviços a serem instaladas e seu modo de operação remete para a compreensão da logística no âmbito de um PSS.

Em um projeto de PSS parte-se da proposição de valor. Considerando o exposto por Morelli (2003), faz-se necessário, no desenvolvimento do PSS, o planejamento da integração entre os diversos atores envolvidos em um PSS, avaliando os possíveis cenários. Na seqüência, a organização ou as organizações envolvidas em um PSS devem se organizar em termos de capacidade requerida. $A$ transição precisa ser estruturada, prevendo o ciclo de vida do PSS.

Nenhuma das referências pesquisadas discutiu diretamente se as dimensões da qualidade tradicionalmente válidas para produtos, sejam bens ou serviços, seriam válidas em um PSS ou se novas dimensões estariam presentes. Nesse sentido, observa-se uma possibilidade de pesquisa. Da mesma forma, medir o grau de aplicação dessas dimensões ou compreender sua importância apresentase como uma oportunidade de continuidade deste trabalho.

Visto que diversos atores podem estar presentes na produção, entrega e pós-venda de um PSS, a consolidação das relações business-to-business (B2B) em parcerias de longo prazo pode ser o elemento que auxilia na relação com o cliente. O processo de comercialização assume novas formas e o papel da força de vendas poderá ser repensado. Em relações B2B, Piercy et al. (1997) já chamavam a atenção para a importância das habilidades e das competências do pessoal de vendas. Além disso, há a necessidade de flexibilidade e autonomia dessa equipe; soma-se a isso a eliminação de barreiras entre diversos departamentos, redução da burocracia e elaboração de um sistema de remuneração compatível com as habilidades e as competências presentes (HOLMLUND, 2008). Nota-se, também, que em muitos casos, uma empresa parceira atua em nome de outra empresa (Borchardt et al., 2008a). A co-responsabilidade e a complexidade das relações é um ponto a ser investigado posteriormente. Da mesma forma, a gestão do conhecimento envolvido nas diferentes categorias de PSS e em todas as organizações participantes pode ser elemento de investigação. 
Ao analisar a questão conceitual acerca de PSS, predominam os conceitos vinculados a sua origem, a ecologia industrial (MANZINI; VEZZOLI, 2003). Mont (2002) destaca, entretanto, que não há consenso entre os autores especialmente quando se trata da abordagem econômica do PSS. Identifica-se, então, como possibilidade de pesquisa a conceituação e caracterização dos diversos tipos de PSS, tanto com relação ao enfoque ambiental como econômico.

Embora autores como Manzini e Vezzoli (2003) enfatizem o impacto positivo em termos ambientais do PSS, os efetivos ganhos ambientais gerados pelos modelos de produção e consumo de bens e serviços, entregues na forma de um PSS ainda são questionáveis (SAKAO et al., 2009). Identifica-se, então, uma oportunidade de investigação que considere, inclusive, o ciclo de vida do PSS (AURICH et al., 2006). Métodos para medição e avaliação de desempenho ambiental têm sido propostos para produção de produtos físicos e serviços (LUZ et al., 2006). Como medir e avaliar o desempenho de um PSS tem um escopo mais amplo e, até onde se investigou, não há trabalhos específicos sobre essa temática.

Para Tukkner e Tischner (2006), um PSS não é inerentemente mais sustentável economicamente que a venda direta de produtos físicos. Um PSS somente será melhor em termos de resultados econômicos se gerar mais valor, tangível e intangível, do que a venda do produto físico (SHARMA et al, 2010). Para tanto, os custos envolvidos em um PSS precisam ser conhecidos e gerenciados e comparados com os ganhos a serem gerados. Esse aspecto pode ser entendido como uma oportunidade de pesquisa.

A relação entre a sustentabilidade ambiental e econômica, mostrada em Borchardt et al. (2010), pode ser pensada em termos das diversas categorias de PSS. Em várias situações, a busca por um melhor desempenho ambiental somente será percebida como algo praticável por parte das organizações se, atrelada a mesma, for percebida a possibilidade de ganhos econômicos (KARLSSON; LUTTROPP, 2006).

Finalizando, o Quadro 4 sumariza as proposições de pesquisa identificadas a partir da análise do referencial teórico construído. 


\begin{tabular}{|l|l|}
\hline \multicolumn{1}{|c|}{ Oportunidade de pesquisa } & \multicolumn{1}{c|}{ Sugestão de pesquisa } \\
\hline Características e conceitos de PSS & $\begin{array}{l}\text { Conceituar e caracterizar os tipos de PSS. } \\
\text { Identificar elementos estratégicos, táticos e operacionais } \\
\text { predominantes em cada categoria de PSS. } \\
\text { Analisar ferramentas de gerenciamento necessárias nas } \\
\text { diversas categorias de PSS }\end{array}$ \\
\hline Logística em PSS & $\begin{array}{l}\text { Estudar os canais de entrega de um PSS; aspectos } \\
\text { relativos a logística de aquisição em um PSS }\end{array}$ \\
\hline $\begin{array}{l}\text { Qualidade e satisfação dos clientes em } \\
\text { PSS }\end{array}$ & $\begin{array}{l}\text { Identificar dimensões da qualidade aplicáveis a um PSS. } \\
\text { Definir método para mensurar a importância e aplicação } \\
\text { dessas dimensões. }\end{array}$ \\
\hline Projeto de PSS & $\begin{array}{l}\text { Analisar e propor métodos de P\&D e projeto de PSS } \\
\text { considerando os diversos atores envolvidos }\end{array}$ \\
\hline Sustentabilidade ambiental do PSS & Avaliar o desempenho ambiental de um PSS \\
\hline Sustentabilidade econômica do PSS & $\begin{array}{l}\text { Identificar possíveis aumentos de margem decorrente da } \\
\text { implantação de um PSS }\end{array}$ \\
\hline Relações B2B em PSS & $\begin{array}{l}\text { Estudar as relações B2B presentes em um PSS, sua } \\
\text { complexidade e papel de cada ator }\end{array}$ \\
\hline
\end{tabular}

Quadro 4 - Sugestões de pesquisas futuras

\section{CONCLUSÕES}

Um dos objetivos deste trabalho foi apresentar o PSS, os tipos existentes, comentar aspectos relacionados ao projeto de um PSS e analisar as vantagens e as dificuldades decorrentes de sua aplicação. Os principais ganhos de um PSS referem-se à sustentabilidade ambiental, à sustentabilidade econômica e à cocriação de valor. Observou-se, no entanto, na literatura pesquisada, que a maior parte dos autores discute conceitos, tipologia e características dos PSS. Não se notou a presença de um quadro de trabalho que possibilite identificar os elementos preponderantes no projeto de cada um dos tipos de PSS apresentado. Da mesma forma, os desafios gerenciais de um PSS não foram tratados nem foi observada uma discussão sobre indicadores de desempenho apropriados para a gestão de um PSS. Outro aspecto abordado diz respeito à análise do ciclo de vida do produto, que passa a englobar a análise do ciclo de vida dos produtos físicos e dos serviços presentes no PSS. Os principais resultados acerca do levantamento bibliográfico realizado são sumarizados no Quadro 5. 


\begin{tabular}{|c|c|}
\hline Item & Descrição \\
\hline $\begin{array}{l}\text { Conceito de } \\
\text { PSS }\end{array}$ & $\begin{array}{l}\text { Mudança de enfoque de produto físico para um sistema integrado de bens } \\
\text { e serviços que visa oferecer soluções para os consumidores. }\end{array}$ \\
\hline $\begin{array}{l}\text { Enfoques do } \\
\text { PSS }\end{array}$ & $\begin{array}{l}\text { Ambiental: possibilita uso mais intensivo dos bens físicos, gerando menos } \\
\text { impacto ambiental; tal modalidade de uso de produtos requer um sistema } \\
\text { de gerenciamento dos mesmos. } \\
\text { Econômico: gera receitas através da relação de longo prazo com o } \\
\text { consumidor, levando a co-criação de valor através do envolvimento de } \\
\text { vários parceiros. }\end{array}$ \\
\hline $\begin{array}{l}\text { Categorias de } \\
\text { PSS }\end{array}$ & $\begin{array}{l}\text { Product-oriented service: } \\
\text { - product-related service } \\
\text { - assessoria e concultoria } \\
\text { Use-oriented service: } \\
\text { - leasing de produtos } \\
\text { - aluguel de produtos / sharing } \\
\text { - product pooling } \\
\text { Result-oriented service } \\
\text { - atividades de gerenciamento e terceirização } \\
\text { - pagamento por unidade de serviço } \\
\text { - resultados funcionais }\end{array}$ \\
\hline $\begin{array}{l}\text { Projeto de um } \\
\text { PSS }\end{array}$ & $\begin{array}{l}\text { Definir atores presentes no PSS; } \\
\text { considerar diversos cenários; } \\
\text { trabalhar com ferramentas de gerenciamento que considerem o PSS como } \\
\text { um todo; } \\
\text { definir a proposição de valor, os riscos, os custos e projetar os ganhos; } \\
\text { projetar a organização em termos de capacidade requerida; } \\
\text { projetar produtos físicos e serviços de forma integrada; } \\
\text { incluir a abordagem ambiental, avaliando impacto em todo o ciclo de vida } \\
\text { do PSS. }\end{array}$ \\
\hline $\begin{array}{l}\text { Fases de } \\
\text { transição de } \\
\text { produtos físicos } \\
\text { para PSS }\end{array}$ & $\begin{array}{l}\text { Fase 1: produtos e serviços são combinados e oferecidos de forma } \\
\text { integrada; } \\
\text { Fase 2: portifólio de produtos é ampliado através da agregação de outros } \\
\text { serviços; } \\
\text { Fase 3: ofertar a entrega de soluções }\end{array}$ \\
\hline $\begin{array}{l}\text { Vantagens do } \\
\text { PSS }\end{array}$ & $\begin{array}{l}\text { Oferecer soluções integradas e customizadas; aumentar a velocidade de } \\
\text { inovação; } \\
\text { construir relações de longo prazo com consumidores e parceiros; } \\
\text { diminuir o impacto ambiental. }\end{array}$ \\
\hline Desafios do PSS & $\begin{array}{l}\text { Consumidores não enxergam benefícios; } \\
\text { dificuldade de projeto devido ao enfoque tradicional e formação dos } \\
\text { projetistas; } \\
\text { cultura do "ter". }\end{array}$ \\
\hline
\end{tabular}

Quadro 5 - Resultados acerca do PSS

O outro objetivo desta pesquisa foi identificar oportunidades de pesquisas relacionadas com PSS. Entre essas, destacam-se: Quais elementos compõem um PSS? Como os mesmos devem ser considerados e analisados por uma empresa que pretende expandir seus produtos através do PSS? Reconhecendo as diversas possibilidades de estruturar um PSS, quais são e como interagem os principais aspectos relacionados à gestão das operações de um PSS? Como mensurar o desempenho de um PSS?

Revista Produção Online, v.10, n.4, p. 837-860, dez., 2010 
Por fim, pela importância observada com relação à rede de parceiros, principalmente na entrega de um PSS, pesquisas relacionadas às relações interorganizacionais são relevantes no contexto do PSS.

Este trabalho mostra-se como uma continuidade das pesquisas efetuadas por Borchardt et al. (2008b), Sellitto et al. (2009) e Sellitto et al. (2010), em que foram estudados aspectos relacionados à integração de serviços de pós-venda em produtos de base tecnológica. A sustentabilidade ambiental através do ecodesign, ou seja, da inserção da análise impacto ambiental ou mesmo da escolha da melhor opção em termos de energia, materiais, canal de distribuição, embalagem, entre outros também conduz ao PSS: o projeto de um PSS, sob o ponto de vista ambiental, poderá fazer uso do ecodesign. Da mesma forma que anteriormente, o PSS pode ser considerado como elemento complementar as pesquisas sobre ecodesign apresentadas em Borchardt et al. (2008b) e Borchardt et al. (2009).

\section{REFERÊNCIAS}

ALMEIDA, L.; MIGUEL, P.; SILVA, M. A literature review of servitization: a preliminary analysis. POMS $-19^{\mathrm{TH}}$. ANNUAL CONFERENCE. PROCEEDINGS OF $19^{\text {TH }}$. ANNUAL CONFERENCE PRODUCTION AND OPERATIONS MANAGEMENT. 2008.

AN, Y.; LEE, S.; PARK, Y. Development of an integrated product-service roadmap with QFD A case study on mobile communications. International Journal of Service Industry Management. v. 19, n. 5, p.621-638, 2008.

AURICH, J. C.; WOLF, N.; Sienes, M.; Schweitzer, E. Configuration of productservice systems. Journal of Manufacturing Technology Management. v. 20, n. 5, p. 591-605, 2009.

AURICH, J. C.; FUCHS, C.; Wagenknecht, C. Life cycle oriented design of technical PSS. Journal of Cleaner Production. v. 14, p.1480-1494, 2006.

AZARENKO, A. et al. Technical product-service systems: some implications for the machine tool industry. Journal of Manufacturing Technology Management, v. 20, n. 5, p. 700-722, 2009.

BAINES, T. et al. State-of-the-art in product-service systems. Proceedings of the Institution of Mechanical Engineers, Part B: Journal of Engineering Manufacture. v.221, n.10, p.1543-1552, 2007. 
Baines, T et al. Towards an operations strategy for product-centric servitization. International Journal of Operations \& Production Management. v. 29, n. 5, p. $494-519,2009$.

BEHARA, R.; GUNDERSEN, D. Analysis of quality management practices in services. International Journal of Quality \& Reliability Management. v. 18, n. 6, p.584-603, 2001.

$\mathrm{BESCH}, \mathrm{K}$. Product-service system for moffice furniture: barriers and opportunities on the European market. Journal of Cleaner Production. v. 13, p. 1083 - 1094, 2003.

BORCHARDT, M.; SELLITTO, M.; PEREIRA, G. Serviços de pós-venda para produtos fabricados em base tecnológica. Revista Produção on Line, v. 8, n. 2, p. 2 $-26,2008$ (a).

BORCHARDT, M.; POLTOSI, L.; SELLITTO, M.; PEREIRA, G. Considerações sobre ecodesign: um estudo de caso na indústria eletrônica automotiva. Revista Ambiente e Sociedade. v. 11, n. 2, p. 1 - 13, 2008 (b).

BORCHARDT, M.; POLTOSI, L.; SELLITTO, M.; PEREIRA, G. Adopting ecodesign practices: case study of a Midsized Automotive Supplier. Environmental Quality Management. v. 19, n. 1, p. $7-22,2009$.

BORCHARDT, M.; WENDT, M.; SELLITTO, M.; PEREIRA, G. Reprojeto do contraforte: um caso de aplicação do ecodesign em manufatura calçadista. Revista Produção. Ahead of print, 2010.

BRAX, S.; JONSSON, K. Developing integrated solution offerings for remote diagnostics: a comparative case study of two manufacturers. International Journal of Operations \& Production Management. v. 29, n. 5, p. 539-560, 2009.

BUNDSCHUH, R.; DEZVANE, T. How to make after sale services pay off. The Mckinsey Quarterly, v.4, n.1, p.116-127, 2003.

COOK, M.; BHAMRA, T.; LEMON, M. The transfer and application of product-service system: from academia to UK manufacturing firms. Journal of Cleaner Production. v. 14, p. $1455-1465,2006$.

EHRENFELD, J. Designing 'Sustainable' Product/Service Systems. 2ND INTERNATIONAL SYMPOSIUM ON ENVIRONMENTALLY CONSCIOUS DESIGN AND INVERSE MANUFACTURING (ECODESIGN'01). Proceedings.Tokyo, Japan, $11-15 / 12,2001$.

GEBAUER, H.; FLEISCH, E. Managing sustainable service improvements in manufacturing companies. Kybernetes. v. 36, n. 5/6, p. 583-595, 2007. 
GEBAUER, $\mathrm{H}$. Identifying service strategies in product manufacturing companies by exploring environment-strategy configurations. Industrial Marketing Management. V. $37,278-291,2008$.

GOEDKOOP, M.; Haler, C.; Riele, H.; Rommers, P. Product Service-Systems, ecological and economic basics. Report for Dutch Ministries of Environment (VROM) and Economic Affairs, Amsterdam, 1999.

HOLMLUND, M. A definition, model, and empirical analysis of business-to-business relationship quality. International Journal of Service Industry Management. v. 19, n. 1, p. 32-62, 2008.

JOHNSTONE, S.; Dainty, A; Wilkinson, A. Integrating products and services through life: an aerospace experience. International Journal of Operations \& Production Management. v. 29, n. 5, p.520-538, 2009.

KANG, M.; WIMMER, R. Product-service system as systemic cures for obese consumption and production. Journal of Cleaner Production. v. 16, p. $1146-1152$, 2008.

KARLSSON, R.; LUTTROPP, C. Ecodesign: what's happening? An overview of the subject area of Ecodesign and the papers in this special issue. Journal of Cleaner Production, v.14, p. 1291 - 1298, 2006.

KIMITA, K.; SHIMOMURA, Y.; ARAI, T. Evaluation of customer satisfaction for PSS design. Journal of Manufacturing Technology Management, v. 20, n. 5, p. 654673, 2009.

KRUCKEN, L.; MERONI, A. Building stakeholder networks to develop and deliver product-service-systems: practical experiences on elaborating pro-active materials for communication. Journal of Cleaner Production, v. 14, p. 1502 - 1508, 2006.

LUZ, S.; SELLITTO, M.; GOMES, L. Medição de desempenho ambiental baseada em método multicriterial de apoio à decisão: estudo de caso na indústria automotiva. Gestão \& Produção, v.13, n.3, p. 557-570, 2006.

MCALOONE, T.; ANDREASEN, M. Design for utility, sustainability and societal virtues: Developing Product Service Systems. INTERNATIONAL DESIGN CONFERENCE (DESIGN 2004). Proceedings. Dubrovnik, 18-21/5, 2004.

MANZINI, E.; VEZZOLI, C. A strategic design approach to develop sustainable product service systems: examples taken from the 'environmentally friendly innovation' Italian prize. Journal of Cleaner Production. v. 11, p. 851-857, 2003.

MONT, O. K.; Clarifying the concept of product-service system. Journal of Cleaner Production, v. 10, p. 237-245, 2002. 
MONT, O.; LINDHQVIST, T. The role of public policy in advancement of productservice system. Journal of Cleaner Production. v. 11, p. $905-914,2003$.

MORELLI, N. Product-service system, a perspective shift for designers: a case study in a design of a telecentre. Design Studies. v. 24, p. $73-99,2003$.

OLIVA, R.; KALLENBERG, R. Managing the transition from products to services. International Journal of Service Industry Management. v. 14, n. 2, p. $160-172$, 2003.

PARASURAMAN, A.; ZEITHALM, V.; BERRY, L. A conceptual model of service quality and its implications for future research. Journal of Marketing. v.49, n.1, p. 41-50, 1988.

PAWAR, K.; BELTAGUI, A.; RIEDEL, J. The PSO triangle: designing product, service and organization to create value. International Journal of Operations \&

Production Management. v. 29, n. 5, p. 468-493, 2009.

PIERCY, N.; CRAVENS, D;. MORGAN, N. Sources of effectiveness in the businessto-business sales organization. Journal of Marketing Practice: Applied Marketing Science. v. 3, n. 1, p. 43-69, 1997.

RAMOS, A.; BORCHARDT, M.; PEREIRA. G. A ponderação das dimensões da qualidade usando a Análise Hierárquica de Processo: um estudo de caso. XXIX ENEGEP. Anais do XXIX Encontro Nacional de Engenharia de Produção. 2009.

RANAWEERA, C.; PRABHU, J. The influence of satisfaction, trust and switching barriers on customers retention in a continuous purchasing set. International Journal of Service Industry Management. v. 14, n. 4, p. 374-395, 2003.

REIJNDERS, L. The factor $X$ debate - setting targets for eco-efficiency. Journal of Industrial Ecology. v.2, n.1, p. 13-21, 1998.

RESE, M.; KARGER, M.; STROTMANN, W. The dynamics of industrial product service systems (IPS2) - using the net present value approach and real pptions approach to improve life cycle management. Journal of Manufacturing Science and Technology. v. 1, p. 279-286, 2009 (a).

RESE, M.; STROTMANN, W.; KARGER, M. Which industrial product service system fits best? Evaluating flexible alternatives based on customers' preference drivers.

Journal of Manufacturing Technology Management. v. 20, n. 5, p. 640-653, 2009 (b).

ROY, R. Sustainable product-service systems. Futures, v. 32, p. $289-299,2000$.

SAKAO, T.; SANDSTRÖM, G.; MATZEN, D. Framing research for service orientation of manufacturers through PSS approaches. Journal of Manufacturing Technology Management. v. 20, n. 5, p. 754-778, 2009. 
SALVADOR, A. Métodos e técnicas de pesquisa bibliográfica. Porto Alegre: Ed. Sulina, 1981.

SELLITTO, M.; BORCHARDT, M.; PEREIRA, G. Análise de temas estratégicos presentes em operações de pós-venda em manufatura de base tecnológica. XVI SIMPEP. Anais do XVI Simposio de Engenharia de Produção, 2009.

SELLITTO, M.; BORCHARDT, M.; PEREIRA, G. Prioridades estratégicas em serviços de pós-venda de uma empresa de manufatura de base tecnológica. Gestão e Produção. v.17, n. 1, p. 95-109, 2010.

Sharma, A.; lyer, G.; Mehrotra, A.; Krishnan, R. Sustainability and business-tobusiness marketing: A framework and implications. Industrial Marketing Management. v. 39, n. 2, p. 330-341, 2010.

SPRING, M.; ARAÚJO, L. Service, services and products: rethinking operations strategy Internacional. Journal of Operations \& Production Management. v. 29, n. 5, p. 444-467, 2009.

TUKKER, A. Eight types of product-service system: eight ways to sustainability? Expiences from Suspronet. Business Strategy and the Environment. V.13, p. 246260, 2004.

TUKKER, A.; TISCHNER, U. Product-services as a research field: past, present and future. Reflections from a decade of research. Journal of Cleaner Production. v. 14, p. $1552-1556,2006$.

VEZZOLI, C.; SCIAMA, D. Experimental educational networking on open research sigues Studying PSS applicability and development in emerging contexts.

International Journal of Sustainability in Higher Education. v. 8, n. 2, p. 198-209, 2007.

VIARDORT, E. Successful marketing for high-tech firms. Norwood : Ed. Artech House, 2004.

WAGNER, S.; ZELLWEGER, T.; LINDEMANN, E. Erfolgreiches after sales service management: durch einen strategie- und lebenszyklusorientierten Ansatz lassen sich potenziale ausschöpfen. Industrie Management, v.23, n.1, p.60-63, 2007.

WILLIANS, A. Product-service sysrem in the automobile industry: contribution to system innovation. Journal of Cleaner Production. v. 15, p. 1093 - 1103, 2007.

YANG, X.; MOORE, P.; PU, J.; WONG, C. A practical methodology for realizing product service systems for consumer products. Computers \& Industrial Engineering. v. 56, p. 224-235, 2009. 
YANAMANDRAM, V.; WHITE, L. Switching barriers in business-to-business services: a qualitative study. International Journal of Service Industry Management. v. 17, n. 2, p. $158-192,2006$.

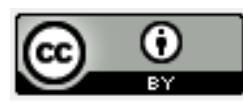

Artigo recebido em 28/07/2008 e aceito para publicação em 24/08/2010. 